\title{
Latent Class Trajectory Analysis of Risk Factors Uncovers Progression to Type 2 Diabetes
}

\author{
Qilu Yu ${ }^{1,3}$, Maurice C. Johnson, Jr. ${ }^{1}$, Howard A. Fishbein ${ }^{*}$, Rebecca J. Birch', Xiaoshu Zhu', Russ Mardon', \\ Wilson Pace ${ }^{2}$, Sunitha M. Mathew' ${ }^{1}$, Holly L. Sawyer ${ }^{1}$, Lori S. Merrill' ${ }^{1}$ Keith D. Umbel', Sophia Jang ${ }^{1}$ \\ Westat, Rockville, MD, USA \\ ${ }^{2}$ DARTNet, Aurora, CO, USA \\ ${ }^{3}$ National Center for Complementary and Integrative Health (NCCIH), National Institutes of Health (NIH), Bethesda, MD, USA
}

\section{Article Info}

\section{Article Notes}

Received: March 19, 2021

Accepted: June 02, 2021

\section{${ }^{*}$ Correspondence:}

*Dr. Howard A. Fishbein, Westat, Rockville, MD, USA;

Telephone No: (443) 812-5710

Email: HowardFishbein@gmail11.com.

${ }^{0} 2021$ Fishbein HA. This article is distributed under the terms of the Creative Commons Attribution 4.0 International License.

\section{Keywords:}

Latent class analysis

Risk factor trajectories

Newly diagnosed type 2 diabetes

Epidemiology

BMI

Blood pressure

Lipids percent changes overtime

Increased risk of disease

\section{Abstract}

We identified trajectories of diabetes risk factors in the Longitudinal Epidemiologic Assessment of Diabetes Risk (LEADR) cohort analyzing 8 years of electronic health records on 1.4 million patients, and investigated associations between trajectories and progression to new onset Type 2 diabetes.

Design and Methods: Analyzing LEADR data (2010-2016), we applied Latent Class Trajectory Analysis (LCTA) to classify patterns of risk factor change. There were 824,043 patients with BMls; 955,128 patients with systolic blood pressures; 957,491 patients with diastolic blood pressures; 300,137 patients with HDLs; 267,553 patients with non-HDL cholesterols; and 297,026 patients with triglycerides. Patients had to have data for all risk factors being assessed. Association between trajectories and incidence of type 2 diabetes for 94,551 patients was assessed using negative binomial regression analysis.

Results: Compared to a static BMI trajectory, those with a sustained weight increase $(25 \%+$ from starting $\mathrm{BMI})$ were at higher risk of type 2 diabetes over 4.8 years of follow-up (range 2.0 to 8.0 years) (adjusted rate ratios ranged 1.531.62 , $p$-value $<0.05$ ). Patients with a BMI decrease trajectory (of $10 \%$ ), were at reduced risk of diabetes (adjusted rate ratios ranged 0.54-0.74, p-value<.05). $\mathrm{BP}$ and lipid trajectories had significant associations with diabetes onset.

Conclusions: Regardless of the starting BMI, those who increased their BMI by $25 \%$ within two years and maintained the higher weight were significantly at increased risk of type 2 diabetes. Monitoring BMI change and other known risk factor trajectories, BP and lipids, are additional tools for identifying patients at risk for type 2 diabetes.

\section{Introduction}

In the United States, diabetes ranks seventh in leading causes of death, and accounts for nearly $\$ 327$ billion in total medical expenditures ${ }^{1}$, with type 2 diabetes mellitus accounting for 90 to 95 percent of all diabetes cases ${ }^{2,3}$. Various factors are associated with the risk of developing type 2 diabetes, including weight, age, race/ethnicity, blood pressure, lipid levels, physical activity, other chronic conditions, and genetics ${ }^{2,4,5}$. The combined effect of these risk factors is a major public health concern, and better management of lifestyle risk factors (e.g., weight, physical activity, diet) may help in addressing the incidence of type 2 diabetes in the United States ${ }^{6,7}$.

Understanding the patterns of potentially modifiable risk factors, such as BMI, blood pressure, and blood lipids, may help in better predicting health outcomes and prevention strategies ${ }^{8}$. Research suggests the relationship between BMI and type 2 diabetes ${ }^{9,10}$, 
altered glucose metabolism ${ }^{11}$, and insulin resistance ${ }^{12}$, is not only due to the presence of BMI above a certain threshold, but also due to changes in BMI over time. A retrospective analysis of nationally representative data estimated 9 percent of diabetes cases could have been prevented had obese individuals decreased their BMI over the 10-year study period ${ }^{13}$. Further, a recent study has suggested the monitoring of change in BMI as an indicator for diabetes screening ${ }^{14}$. While recent studies have examined the overall pattern of BMI levels over time ${ }^{15,16,17}$, few studies to our knowledge have explored the changing patterns of BMI and their relationship to type 2 diabetes ${ }^{16}$. In addition, few studies have examined the potential impact of blood pressure and serum lipid trajectories on risk of developing type 2 diabetes ${ }^{18,19,20,21}$.

Using electronic health records (EHR) from the Longitudinal Epidemiologic Assessment of Diabetes Risk (LEADR) cohort $^{22}$, we examined the trajectories of multiple risk factors for type 2 diabetes including: BMI, serum triglycerides, HDL cholesterol, non-HDL cholesterol, systolic (SBP) and diastolic blood pressure (DBP). We then investigated the associations between the trajectories and development of new onset type 2 diabetes.

\section{Research Design and Methods}

\section{Study Population}

The LEADR cohort is a dynamic database consisting of patients aged $\geq 18$ years from nine participating healthcare networks across the United States who have at least four encounters with the healthcare system, each occurring at least 14 days apart, and at least 24 months between the first and last encounters (an encounter is defined as individual times when either one or more conditions, observations, procedures, drug records, or visit records occurred). To identify incident cases of type 2 diabetes, those with type 1 diabetes, type 2 diabetes, gestational diabetes, or unspecified diabetes at cohort entry or during their first 12 months in the cohort were excluded. This analysis used the first wave of the LEADR cohort, which had 1.4 million patients seen between January 1, 2010, and December 31, 2016.

In addition to the LEADR cohort inclusion criteria, for the trajectory analysis, patients were required to have at least two measures for each one of the above noted predefined risk factors $\geq 14$ days apart; sample sizes for each high-risk factor trajectory analysis differed based on availability of clinical measurements for patients. The resulting patient sample size for risk factors were: BMI 824,043, SBP 955,128, DBP 957,491, HDL 300,137, nonHDL 267,553, and triglycerides 297,026 . To meet the requirement to have all risk factor trajectories for type 2 diabetes generated, to be included in the study, the result was 94,551 LEADR patients met this criterion.

\section{Trajectories of High-Risk Factors}

Using latent class trajectory analysis (LCTA), we classified patients according to their high-risk factor patterns over time. For each selected risk factor, we examined actual change in measurements over time. Measures were grouped into 6-month windows starting from the date of first measure with a maximum of up to 10 windows, totaling 5 years of follow-up. For each risk factor, the mean risk factor value with the 6-month windows were used in the analysis, with the mid-date of the 6-month windows assigned to be the corresponding time score. When latent class trajectories differed only in level, and failed to differentiate on patterns of change, we repeated the analysis on percentage change from baseline over time. This occurred only for BMI, for which an additional LCTA was conducted on percentage change (defined as [BMI_at observed timepoint- BMI_baseline)/ BMI_baseline]). We also looked at age and sex differences in trajectories to identify differential patterns in risk factor dynamics.

Using the software Mplus (version 8.2) ${ }^{23}$, we conducted latent class trajectory analysis (LCTA) with 3-, 4-, 5- and 6-classes, allowing intercept, slope and quadratic terms to vary across classes, but not within classes (i.e., individuals were grouped into latent classes with the assumption that everyone in the group followed the same trajectory). The final number of classes and the best-fit model for each trajectory were determined by comparing Akaike's Information Criterion (AIC), Bayesian Information Criterion (BIC), entropy, and bootstrapped likelihood ratio test results. In addition, the resulting classes for each model were required to have at least 0.5 percent of the study sample represented. Once the classes from the bestfit model for each risk factor were established, a nominal categorical variable, i.e., class membership, was created to describe the trajectory membership of each patient for each risk factor. Each patient was assigned to the trajectory class for which they had the highest posterior probability of membership. The latent class trajectory curves were plotted using estimated sample means within each sex and/or age stratum.

Based on the observed differences in trajectory patterns for each risk factor, the final BMI and percentage change in BMI trajectories were stratified for males and females by age group, (18-44, 45-64, and 65 years and older, respectively). For blood pressure (SBP and DBP) trajectories, the analysis was only stratified by age group, as further stratification by sex did not reveal substantial differences in the trajectory patterns between males and females. For serum lipids, the trajectory patterns among sex and age groups were similar, therefore these trajectories were not stratified. 


\section{New Onset of Type 2 Diabetes}

Type 2 diabetes was defined using a combination of diagnosis records, prescription drugs, and lab values. These were: 1) diagnosis of type 2 diabetes or unspecified diabetes made on two dates within 24 months (diagnosis dates $\geq 14$ days apart), or 2 ) a prescription for metformin or glucagon-like peptide-1 (GLP1) agonists and a type 2 diabetes or unspecified diabetes diagnosis on any encounter, or 3) a prescription for an antidiabetic agent, or 4 ) hemoglobin A1c lab result $\geq 6.5 \%$ or fasting plasma glucose $\geq 126 \mathrm{mg} / \mathrm{dl}$, or random glucose $\geq 250 \mathrm{mg} / \mathrm{dl}$.

\section{Modeling Development of Type 2 Diabetes}

Negative binomial regression analysis was applied to investigate the relationship between risk factor trajectories and type 2 diabetes. In addition to the trajectories, the preliminary model assessed the association of the following co-variables with diabetes (yes/no) in the multivariable setting: age group (18 to 44 years, 45 to 64 years, and 65 and older), race/ethnicity (non-Hispanic white, nonHispanic black, Hispanic, Asian/AI/AN/Other/Multiple races), sex, geographic region, usage of medicines for hypertension and hyperlipidemia, cardiovascular disease, depression, family history of diabetes, history of tobacco use, number of encounters, and first-recorded BMI. The health history of the study population was derived from the Observational Medical Outcomes Partnership (OMOP) vocabulary and descriptions ${ }^{24}$. Number of health system encounters by the patient was transformed so the model estimate represents a 10-unit increase in number of encounters (number of encounters/10). We assessed interactions between trajectories of risk factors and age group and sex. Variables and interaction terms with a $\mathrm{p}$-value of less than 0.10 were included in the final model. The negative binomial dispersion parameter was estimated by maximum likelihood, and the dispersion parameter was estimated to be $<0.0001$, indicating lack of over-dispersion.

The primary independent variables used in the final negative binomial model for development of type 2 diabetes were trajectory classes of SBP, DBP, HDL, non-HDL, triglycerides, percentage change in BMI, and first recorded BMI. Other variables adjusted for in the model included age group, sex, race/ethnicity, region, first recorded BMI, number of encounters, history of tobacco use, and family history of diabetes. Depression, antihypertension medication use, and lipid lowering medication use were not included in the final model because the respective interaction $\mathrm{p}$-values were greater than 0.10 .

\section{Results}

\section{Patient Characteristics}

Table 1 presents a summary of the demographic and clinical characteristics of the analytic population at baseline for the 94,551 patients who met the cohort inclusion criteria for the LCTA analysis. Of these, 10,785 patients (11.4\%) were diagnosed with type 2 diabetes during a median follow-up of 4.8 years (range 2.0-8.0 years). The study population was $77.8 \%$ non-Hispanic white, $18.8 \%$ Hispanic, and 3.4\% were either non-Hispanic black, Asian, American Indian/Native American, or other racial/ethnic group. Half of the patients were 45 to 64 years, with the rest about evenly split between 18 to 44 years (25.6\%) and 65 and older (23.8\%). In addition, $17.8 \%$ of the population had cardiovascular disease, $25.3 \%$ had depression,

Table 1: Characteristics of the LEADR Study Analytic Population at Baseline

\begin{tabular}{|c|c|}
\hline Characteristic & N (\%) \\
\hline Total Population & $94,551(100)$ \\
\hline Diabetes Onset & $10,785(11.4)$ \\
\hline \multicolumn{2}{|l|}{ Race/Ethnicity } \\
\hline Non-Hispanic White & $73,520(77.8)$ \\
\hline Hispanic & $17,818(18.8)$ \\
\hline $\begin{array}{l}\text { Other (Non-Hispanic Black, Asian, Al/AN, Other, } \\
\text { Multiple) }\end{array}$ & $3,213(3.4)$ \\
\hline \multicolumn{2}{|l|}{ Age in 2010} \\
\hline $18-44$ & $24,205(25.6)$ \\
\hline $45-64$ & $47,848(50.6)$ \\
\hline 65 and older & $22,498(23.8)$ \\
\hline \multicolumn{2}{|l|}{ Sex } \\
\hline Male & $41,074(43.4)$ \\
\hline Female & $53,477(56.6)$ \\
\hline \multicolumn{2}{|l|}{ Health System Regions } \\
\hline Rocky & $53,827(56.9)$ \\
\hline South & $10,436(11.0)$ \\
\hline West & $30,288(32.0)$ \\
\hline Antihypertensive Medication Use & $46,705(49.4)$ \\
\hline Lipid-Lowering Medication Use & $35,729(37.8)$ \\
\hline Cardiovascular Disease & $16,849(17.8)$ \\
\hline Depression & $23,903(25.3)$ \\
\hline History of Tobacco Use & $25,772(27.3)$ \\
\hline Family History of Diabetes & $1,757(1.9)$ \\
\hline Characteristic & Mean (SD) \\
\hline Body Mass Index, first measurement $\left(\mathrm{kg} / \mathrm{m}^{2}\right)$ & $28.5(6.3)$ \\
\hline Number of Encounters & $38.6(25.1)$ \\
\hline Follow-Up (years) & $4.6(1.8)$ \\
\hline Average Body Mass Index $\left(\mathrm{kg} / \mathrm{m}^{2}\right)$ & $28.6(6.2)$ \\
\hline Average Systolic BP (mm Hg) & $126.3(11.7)$ \\
\hline Average Diastolic BP (mm Hg) & $76.4(7.1)$ \\
\hline Average Serum Triglycerides* $(\mathrm{mg} / \mathrm{dL})$ & $141.7(82.8)$ \\
\hline Average Serum HDL (mg/dL) & $51.6(14.7)$ \\
\hline Average Serum Non-HDL (mg/dL) & $136.5(34.8)$ \\
\hline
\end{tabular}

*Serum triglycerides has a right-skewed distribution. The median value is 122.5 .

NOTE: $\mathrm{BP}=$ blood pressure; $\mathrm{N}=$ number.

Patients included in this analysis had at least two measurements for each trajectory. variable, occurring at least 14 days apart (BMI, SBP, DBP, serum triglycerides, serum HDL, and serum non-HDL). 
and $27.3 \%$ reported a history of tobacco use. Half the population were on antihypertensive medications (49.4\%) and $37.8 \%$ on lipid-lowering medications. Mean BMI first recorded was $28.5 \mathrm{~kg} / \mathrm{m}^{2}$. Average number of encounters was 38.6 and the mean follow-up time was 4.6 years. Mean values for each of the risk factors are shown in Table 1.

\section{Trajectories of High-Risk Factors}

\section{BMI}

For the BMI trajectories, we first examined absolute BMI trajectories by sex and age (18 to 44 years, 45 to 64 years, and 65+ years). Five classes emerged from the initial analysis of raw BMI values, with two stable classes representing the vast majority of patients (results not shown). One class exhibited patients who were normal to slightly overweight throughout the study period (BMI range of 22 to $27 \mathrm{~kg} / \mathrm{m}^{2}$ across stratum), and the other class represented patients who were overweight to slightly obese (BMI range of 28 to $32 \mathrm{~kg} / \mathrm{m}^{2}$ across stratum). Together, these two classes represented 85 to $90 \%$ of patients for each BMI sex and age stratum, suggesting most patients in the LEADR cohort had a static weight pattern throughout their time in the cohort. To further differentiate patterns of change in BMI, we therefore repeated the analysis on percentage change in BMI from baseline $(\triangle \mathrm{BMI})$, enabling identification of unique patterns of weight change regardless of a patient's starting BMI.

Each class from the $\triangle B M I$ analyses had patients with normal, overweight, or obese weights at baseline. Figure $1 \mathrm{~A}-\mathrm{F}$ presents the five trajectory classes resulting from

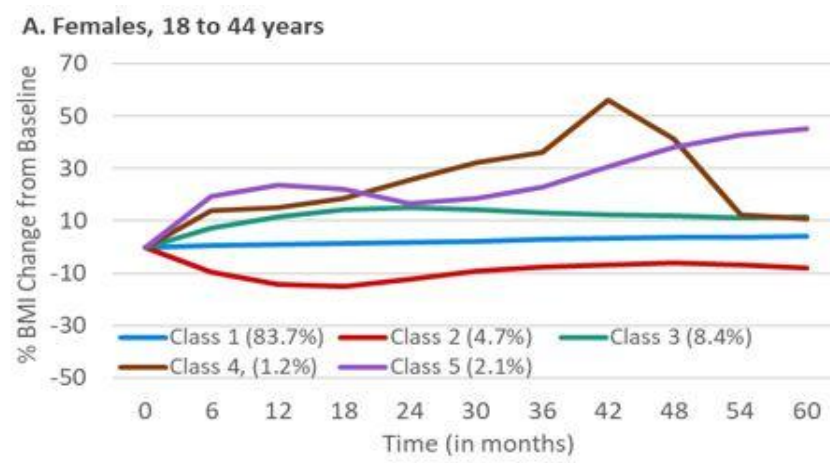

B. Females, 45 to 64
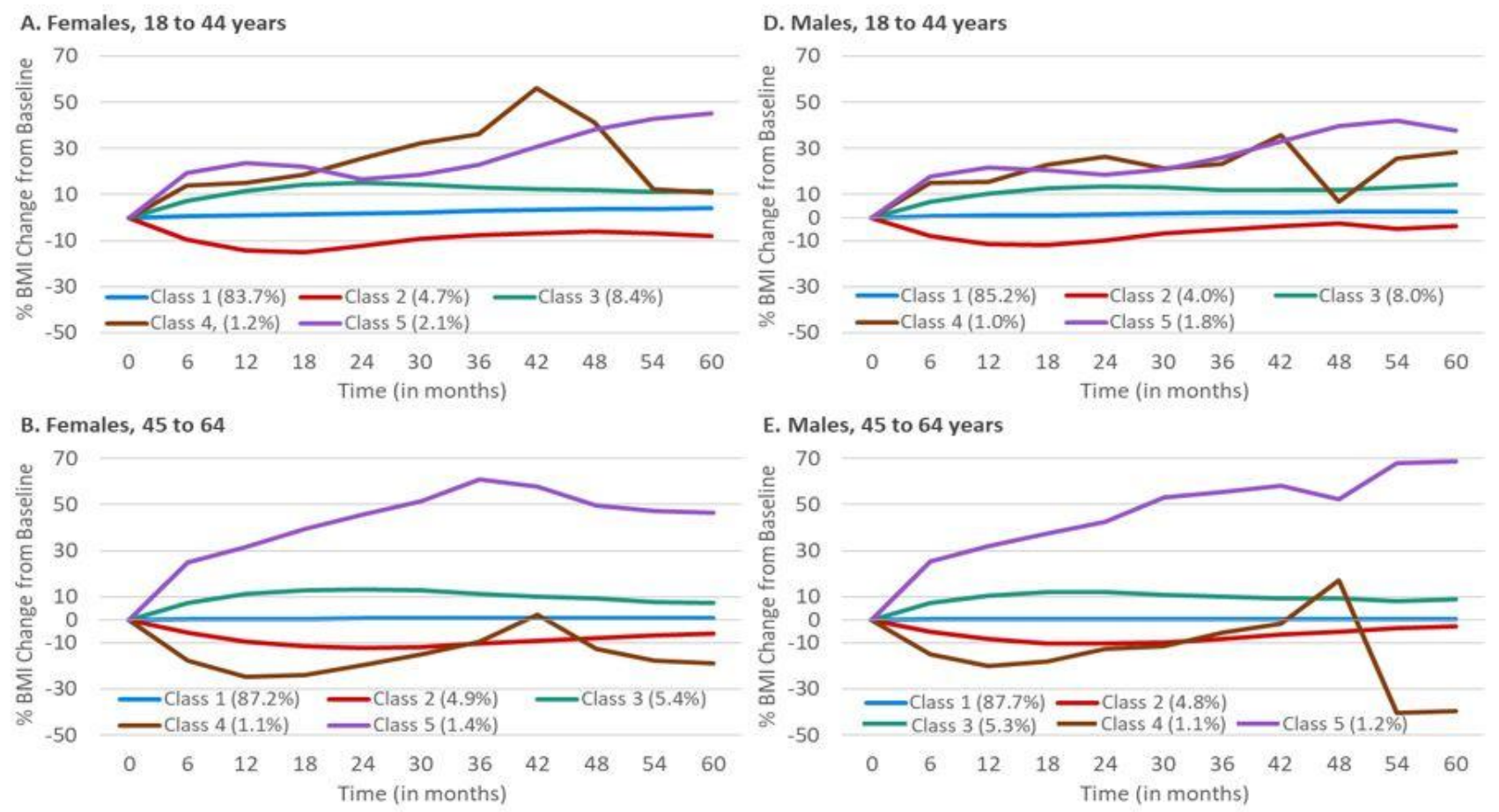

E. Males, 45 to 64 years

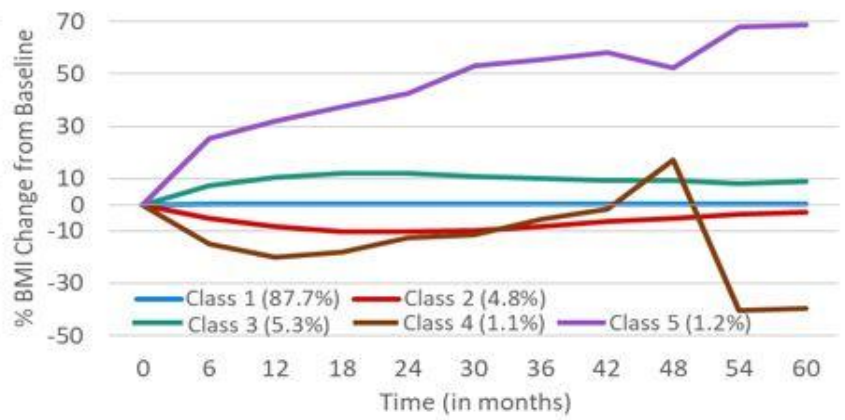

C. Females, 65 years and older
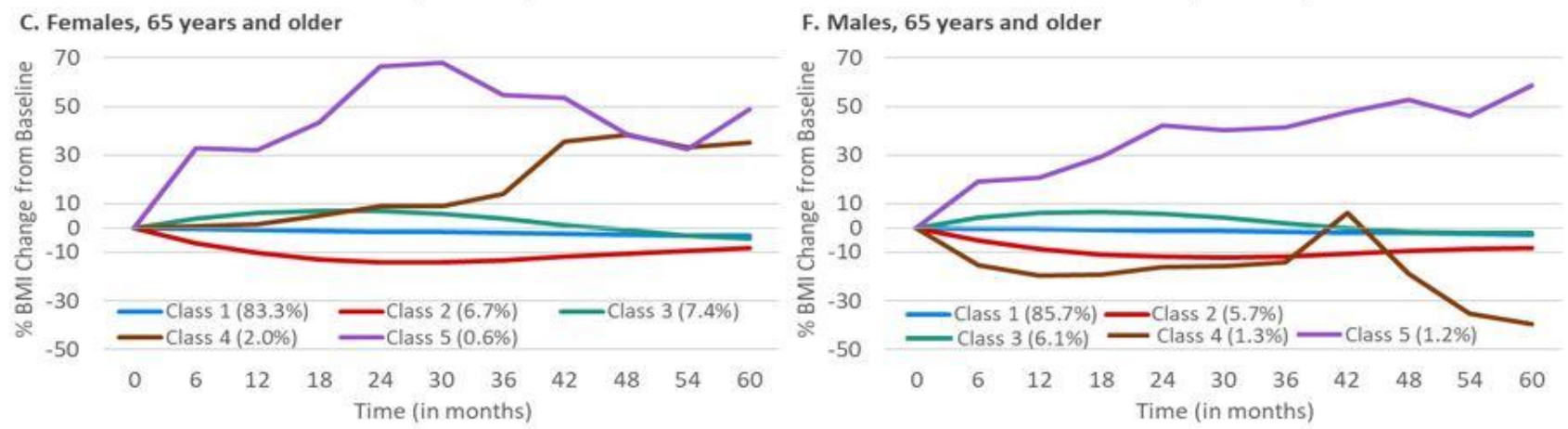

NOTE: Trajectories during 5 years of follow-up until diagnosis of type 2 diabetes, or censoring from the study ( $x$-axis). Mean risk-factor value and the median date of the 6-month windows were used to identify distinct trajectories of change over time. A-female age 18-44 $(n=228,146)$, B-female age 45-64 ( $n=190,350), C$-female age 65+ (81,350), D-male age 18-44 ( $n=122,662), E-m a l e ~ a g e ~ 45-65$ ( $n=139,125)$, F-male age $65+(n=62,410)$. The largest proportion of the patients for each strata was assigned $\Delta B M I$ Class 1 , representing static weight change.

Figure 1: Trajectory of Percentage Change in BMI $(\triangle B M I)$ by Sex and Age Group for LEADR Cohort Patients 
$\triangle B M I$ for each sex and age stratum. Sample size and percentage of the population in each class are presented in Supplemental Table 1. $\triangle \mathrm{BMI}$ Class 1 was defined by patients in all six strata who had a percentage change in BMI near zero over time (i.e., "static weight"). This class corresponds to the two large classes in the absolute BMI trajectory analysis. Patients in $\triangle \mathrm{BMI}$ Class 2 were defined by showing an initial decrease in BMI of near 10 percent, with some strata showing a reversion to their baseline BMI over the study period (i.e., "slight weight loss"). Patients in $\triangle \mathrm{BMI}$ Class 3 were defined by showing an initial increase in BMI nearing 10 percent, then trending back toward their baseline BMI (i.e., "slight weight gain"). Specific patterns of change over time varied across strata for defining patients in $\triangle \mathrm{BMI}$ Class 4 ; in all strata for $\Delta \mathrm{BMI}$ Class 4 the patients' percentage change in BMI generally showed greater fluctuation compared to that of patients in $\triangle \mathrm{BMI}$ classes 1,2 , and 3 (i.e., "fluctuating weight"). $\Delta$ BMI Class 5 was defined by patients who presented with a sharp increase in BMI from baseline of nearly 25\% within 12 months and even more weight gain by the end of the follow-up period (i.e., "sustained weight increase").

\section{Systolic and Diastolic Blood Pressure}

LCTA stratified by age, resulted in three distinct classes for SBP and DBP for each age group (Figure 2 A-F). The sample size and percentage of the population in each class is presented in Supplemental Table 2. With the exception of DBP for those ages 18-44, the blood pressures exhibited similar trajectory patterns. Class 1 represented $89.1 \%$ to $97.5 \%$ of patients in each stratum and presented relatively "static normal blood pressures." The main difference across stratum was the baseline blood pressures for both systolic and diastolic blood pressures; the mean SBP ranged from $120 \mathrm{~mm} \mathrm{Hg}$ to 130 $\mathrm{mm} \mathrm{Hg}$, and the mean DBP was approximately $75 \mathrm{~mm} \mathrm{Hg}$. Class 2 blood pressures generally presented "negative parabolic" patterns, where blood pressures initially increased, reached a point, and then decreased over time. Conversely, Class 3 presented "positive parabolic" patterns, where blood pressures decreased initially, reached a point, and then increased over time. The mean SBP readings for SBP Classes 2 and 3 remained above recommended levels throughout the study period for SBP, ranging from $127 \mathrm{~mm} \mathrm{Hg}$ to $160 \mathrm{~mm} \mathrm{Hg}$; DBP values ranged from $64 \mathrm{~mm} \mathrm{Hg}$ to $94 \mathrm{~mm} \mathrm{Hg}^{25}$.

As previously noted, the stratum that showed a differing trajectory pattern was the youngest age group (18-44 years) for DBP. DBP Class 1 for this stratum was similar to the other strata, presenting a relatively static measurement throughout the study period ( $\sim 73 \mathrm{~mm} \mathrm{Hg})$. For DBP Class 2 patients presented a slowly decreasing diastolic blood pressure, starting from $\sim 86 \mathrm{~mm} \mathrm{Hg}$ and ending at $\sim 81 \mathrm{~mm}$ Hg. DBP Class 3 was defined by a slight up and down pattern, but generally stayed near 85 mm Hg throughout the study period.

\section{Lipids}

HDL analysis resulted in 4 Classes being defined. (Figure $2 \mathrm{G}$ ). The sample size and percentage of the population in each class is presented in Supplemental Table 2. For HDL Class 1, about two-thirds (67\%) of the patients had HDL levels that increased slightly over time from $46.8 \mathrm{mg} / \mathrm{dL}$ to $49.5 \mathrm{mg} / \mathrm{dL}$. This class had the lowest HDL levels of all four classes. For HDL Class 2, 19.2 percent of patients showed a slight convex-shaped trajectory over time with mean values around $68 \mathrm{mg} / \mathrm{dL}$. Patients in HDL Class 3 (12.1\%) showed a concave-shaped trajectory over time with mean values around $71 \mathrm{mg} / \mathrm{dL}$. Lastly, in HDL Class 4, 1.9 percent of patients started with the highest concentrations $(90 \mathrm{mg} /$ $\mathrm{dL}$ ), and had the most change over time.

Four LCTA classes were identified for non-HDL measures (Figure 2H). The sample size and percentage of the population in each class is presented in Supplemental Table 2. Non-HDL Class 1 represented the largest percentage of the cohort (87\%) and had consistent concentrations of $130 \mathrm{mg} / \mathrm{dl}$ over the study period. The remaining 13 percent of patients, split into two classes, showed nonlinear change over time and starting concentrations above recommended values ${ }^{26}$.

Five LCTA classes were identified for triglyceride measures (Figure 2I). The sample size and percentage of the population in each class is presented in Supplemental Table 2. The majority of individuals in the study (83\%) had consistent triglyceride concentrations across the study period. These patients were split into two classes, one with mean values around $90 \mathrm{mg} / \mathrm{dL}$ (Tri Class 1,50\%) and the other with mean values around $148 \mathrm{mg} / \mathrm{dL}$ (Tri Class $2,33 \%$ ). The remaining proportion of patients did show change over time in their triglyceride concentrations. The mean baseline concentrations in Tri Class 3 (6.6\%) were $170 \mathrm{mg} / \mathrm{dL}$, which then increased to $265 \mathrm{mg} / \mathrm{dL}$ at 30 months, then decreased to $196 \mathrm{mg} / \mathrm{dL}$. The mean baseline concentrations in Tri Class 4 (8.6\%) were $280 \mathrm{mg} / \mathrm{dl}$, which then decreased to $187 \mathrm{mg} / \mathrm{dl}$ at 24 months, then increased to $237 \mathrm{mg} / \mathrm{dl}$ at the end of the study. Patients in Tri Class 5 $(2.2 \%)$ had the highest concentrations at about $400 \mathrm{mg} / \mathrm{dL}$ throughout the study period.

\section{Type 2 Diabetes Development}

Table 2 shows the rate ratios (RR) (as exponential of the estimated coefficients) and 95\% CI of all predictors of progression to diabetes based on the final negative binomial model. Given that nominally equivalent latent classes sometimes appear to meaningfully differ in their estimated mean trajectories across sex/age strata, interactions of age and sex with class were included in 

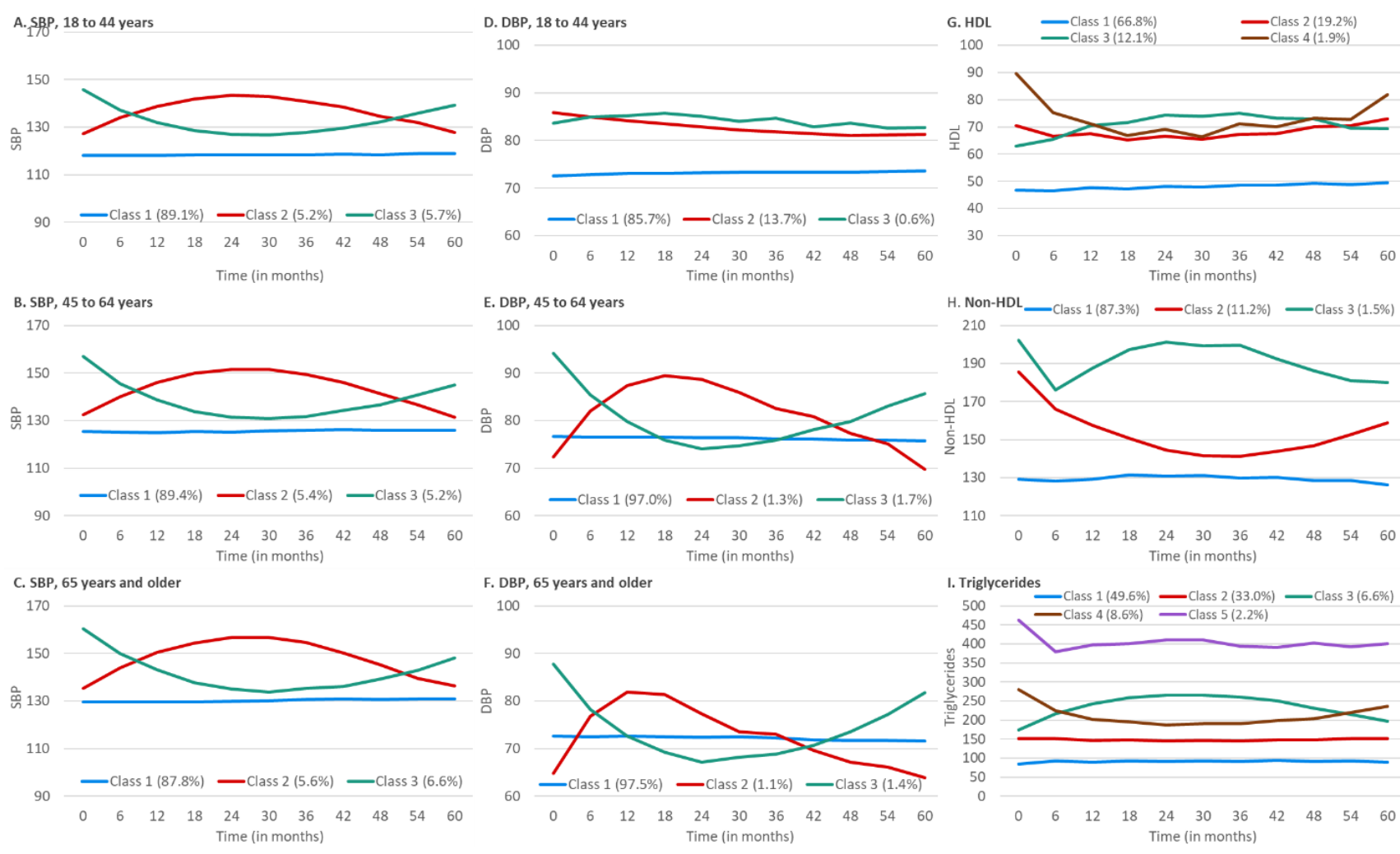

NOTE: Trajectories for systolic and diastolic blood pressures (SBP and DBP) (y-axis mm Hg); and HDL, non-HDL, and triglycerides (Trig) ( $y$-axis $\mathrm{mg} / \mathrm{dL}$ ), during 5 years of follow-up (x-axis) until diagnosis of type 2 diabetes, or censoring from the study. Mean risk-factor value and the median date of the 6-month windows were used to identify distinct trajectories of change over time. A-SBP age 18-44 $(n=421,327), B-S B P$ age $45-64(n=364,216), C-S B P$ age $65+(169,582), D-D B P$ age $18-44(n=422,348), E-D B P$ age $45-65$ ( $n=365,230), F-D B P$ age $65+(n=169,913)$; G-HDL $(n=300,137), H-n o n-H D L(n=267,553)$, and I-Trig $(N=297,026)$. The largest proportion of the patients for each respective risk factor strata was assigned Class 1, representing static blood pressure or lipid value throughout study period.

Figure 2: Trajectories of Blood Pressures (Systolic and Diastolic) by Age Group and Trajectories of Lipids (HDL, non-HDL, and Triglycerides) for LEADR Cohort Patients.

all models and stratified results are presented whenever significant interactions are identified.

For trajectories of SBP measurements, compared to patients with a static trajectory pattern (SBP Class 1), classes with parabolic SBP trajectory patterns, (SBP Classes 2 and 3), were associated with higher risks of developing diabetes, (Adjusted RR [95\% CI], 1.45 [1.30, 1.62] for SBP Class 2 and $1.42[1.28,1.56]$ for Class 3). Results were similar for DBP, 1.34 [1.21, 1.48] for DBP Class 2 and $1.23[0.97,1.55]$ for DBP Class 3, when compared to DBP 1. However, the difference in diabetes risk was not statistically significant ( $p$-value $=0.09$ ) between DBP Class 3 and DBP Class 1 for DBP.

Higher levels of HDL were associated with a reduced risk of diabetes (HDL Classes 2 and 3 compared to 1), however, those in HDL Class 4 who started very high, but then decreased rapidly showed an increased risk of diabetes compared to HDL Class $1(1.26[1.12,1.62])$. For serum triglyceride trajectories, as levels increased, the risk of diabetes increased. Differences in diabetes risk between trajectories of non-HDL were not significant.
Due to the interaction of the change in BMI trajectories and age in the model, rate ratios for BMI change trajectories are shown stratified by age group. For all age groups, those with a slight weight loss trajectory $(\Delta \mathrm{BMI}$ Class 2), had a reduced risk of diabetes compared to patients with static weight trajectory $(\triangle \mathrm{BMI}$ Class 1 ) (18 to 44 years $0.74[0.57,0.96], 45$ to 64 years $0.66[0.55,0.79]$, and 65 years and older $0.54[0.41,0.72])$. The slight weight gain and fluctuating weight trajectories $(\triangle \mathrm{BMI}$ Classes 3 and 4), were not statistically different from $\Delta B M I$ Class 1 in any age group. The sustained weight increase trajectory ( $\triangle \mathrm{BMI}$ Classes 5) had an increased risk of diabetes (18 to 44 years 1.63 [1.12, 2.22], 45 to 64 years 1.53 [1.23, 1.91], and 65 years and older 1.62 [1.12, 2.36]).

Baseline BMI was one of the most significant predictors of diabetes, after adjusting for all other variables in the model, with a $p$-value $<0.0001$. Each one-unit increase in BMI, yielded an increased risk of 1.05 (Table 2). Thus, an individual with a baseline BMI of $35 \mathrm{~kg} / \mathrm{m}^{2}$ has a 64 percent greater risk of diabetes compared to an individual with a baseline BMI of 25 $\mathrm{kg} / \mathrm{m}^{2}$, holding all other variables the same. 
Table 2: Adjusted Rate Ratios and $95 \% \mathrm{Cl}$ from Negative Binomia Model Assessing Risk of Developing Diabetes for LEADR Cohort Patients

\begin{tabular}{|c|c|c|}
\hline Predictor & Rate Ratio $(95 \% \mathrm{Cl})$ & p-value \\
\hline \multicolumn{3}{|l|}{ Systolic Blood Pressure Trajectory } \\
\hline Class 2 vs. 1 & $1.45(1.30,1.62)$ & $<.0001$ \\
\hline Class 3 vs. 1 & $1.42(1.28,1.56)$ & $<.0001$ \\
\hline \multicolumn{3}{|l|}{ Diastolic Blood Pressure Trajectory } \\
\hline Class 2 vs. 1 & $1.34(1.21,1.48)$ & $<.0001$ \\
\hline Class 3 vs. 1 & $1.23(0.97,1.55)$ & 0.09 \\
\hline \multicolumn{3}{|l|}{ Serum HDL Trajectory } \\
\hline Class 2 vs. 1 & $0.69(0.64,0.75)$ & $<.0001$ \\
\hline Class 3 vs. 1 & $0.75(0.68,0.83)$ & $<.0001$ \\
\hline Class 4 vs. 1 & $1.26(1.12,1.43)$ & 0.0002 \\
\hline \multicolumn{3}{|l|}{ Serum Non-HDL Trajectory } \\
\hline Class 2 vs. 1 & $0.95(0.88,1.01)$ & 0.10 \\
\hline Class 3 vs. 1 & $1.05(0.91,1.21)$ & 0.53 \\
\hline \multicolumn{3}{|l|}{ Serum Triglyceride Trajectory } \\
\hline Class 2 vs. 1 & $1.43(1.37,1.50)$ & $<.0001$ \\
\hline Class 3 vs. 1 & $1.92(1.78,2.07)$ & $<.0001$ \\
\hline Class 4 vs. 1 & $1.88(1.76,2.00)$ & $<.0001$ \\
\hline Class 5 vs. 1 & $2.49(2.25,2.76)$ & $<.0001$ \\
\hline \multicolumn{3}{|l|}{ Age and \% BMI Change Trajectory } \\
\hline \multicolumn{3}{|l|}{ Age 18 to 44 years } \\
\hline BMI Class 2 vs. 1 & $0.74(0.57,0.96)$ & 0.02 \\
\hline BMI Class 3 vs. 1 & $0.90(0.73,1.11)$ & 0.33 \\
\hline BMI Class 4 vs. 1 & $1.35(0.93,1.96)$ & 0.12 \\
\hline BMI Class 5 vs. 1 & $1.63(1.12,2.22)$ & 0.002 \\
\hline \multicolumn{3}{|l|}{ Age 45 to 64 years } \\
\hline BMI Class 2 vs. 1 & $0.66(0.55,0.79)$ & $<.0001$ \\
\hline BMI Class 3 vs. 1 & $1.09(0.95,1.26)$ & 0.20 \\
\hline BMI Class 4 vs. 1 & $0.92(0.70,1.21)$ & 0.57 \\
\hline BMI Class 5 vs. 1 & $1.53(1.23,1.91)$ & 0.0002 \\
\hline \multicolumn{3}{|l|}{ Age 65 years and older } \\
\hline BMI Class 2 vs. 1 & $0.54(0.41,0.72)$ & $<.0001$ \\
\hline BMI Class 3 vs. 1 & $0.96(0.77,1.20)$ & 0.71 \\
\hline BMI Class 4 vs. 1 & $0.69(0.45,1.06)$ & 0.09 \\
\hline BMI Class 5 vs. 1 & $1.62(1.12,2.36)$ & 0.01 \\
\hline \multicolumn{3}{|l|}{ Sex } \\
\hline Female vs. Male & $1.07(1.03,1.11)$ & 0.001 \\
\hline \multicolumn{3}{|l|}{ Race/Ethnicity } \\
\hline Hispanic vs. Non-Hispanic White & $1.10(1.03,1.17)$ & 0.002 \\
\hline Other vs. Non-Hispanic White & $1.35(1.24,1.46)$ & $<.0001$ \\
\hline \multicolumn{3}{|l|}{ Region } \\
\hline Rocky vs. West & $0.51(0.48,0.55)$ & $<.0001$ \\
\hline South vs. West & $1.59(1.49,1.69)$ & $<.0001$ \\
\hline Baseline BMI & $1.051(1.048,1.053)$ & $<.0001$ \\
\hline History of tobacco use & $1.15(1.09,1.22)$ & $<.0001$ \\
\hline Family history of diabetes & $1.33(1.19,1.49)$ & $<.0001$ \\
\hline Number of Encounters (10 unit change) & $1.122(1.115,1.129)$ & $<.0001$ \\
\hline
\end{tabular}

Note: Sample size is 94,551 patients. The largest proportion of the patients for each risk factor trajectory was assigned Class 1, generally representing a static $\mathrm{BMI}$, blood pressure, or lipid value

Each of the adjusted variables had a significant relationship with diabetes. Compared to males, females were at greater risk of developing diabetes $(1.07$ [1.03, 1.11]). Likewise, those with history of tobacco (1.15 [1.09, $1.22])$, family history of diabetes $(1.33[1.19,1.49])$, and increasing number of encounters $(1.12[1.12,1.13])$ were also at increased risk of diabetes. While there was no significant difference was found between Hispanic and Non-Hispanic White individuals, those classified as Other were significantly more likely to develop diabetes $(1.35$ $[1.24,1.46])$. Last, the rate ratio ranged from $0.51(0.48$, $0.55)$ to $1.59(1.49,1.69)$, indicating the healthcare system the patient received care influenced risk of developing diabetes.

\section{Conclusions}

By applying a LCTA approach to a large EHR cohort, we identified classes with similar underlying trajectories of longitudinal percentage change in BMI, blood pressure, and lipids that may be helpful in managing a patient's type 2 diabetes risk. Trajectory groups indicating an increased risk of type 2 diabetes included 1) patients with blood pressures in hypertensive range for at least one 6-month time period, 2) patients with triglyceride levels consistently at or above $150 \mathrm{mg} / \mathrm{dL}$ throughout their time in the cohort, and 3) patients with sustained weight increase throughout their time in the cohort. In addition, slight decreases in BMI over time was associated with reduced risk. Our findings emphasize the importance of monitoring weight, blood pressure, and serum lipids over time to identify patients at risk for type 2 diabetes. Furthermore, the potential value of tracking percentage change in BMI from baseline over time for a patient may provide additional information about their risk of progression to type 2 diabetes.

Results from our trajectory analyses identified patterns of physical and clinical laboratory measures that can be used to evaluate a patient's risk for developing type 2 diabetes. Chief among risk reduction is weight control as reflected in BMI measures. For example, like previous studies, our study shows that after controlling for various risk factors, increasing BMI raises the risk for type 2 diabetes $(12,16)$. Among the trajectories, the highest rate ratios associated with diabetes was the class associated with an initial increase in BMI of at least 25\% within two years and maintenance of the increased weight (i.e., sustained weight increase). Our study also found patients with an approximate 10 percent increase in BMI during their first two years in the study, but with a gradual return to baseline weight moderated the risk of diabetes onset, after adjusting for baseline BMI. Conversely, patients with an approximate 10 percent decrease in BMI, showed a significant reduction in risk of diabetes onset, after adjusting for baseline BMI. Our results align with previous studies indicating weight management is an effective strategy for reducing the risk of type 2 diabetes ${ }^{27,28}$. 
We identified three distinct patterns for SBP and DBP measures overtime. For both trajectory models that crossed acceptable hypertension reading levels, our data confirmed other research findings, that hypertension is a significant risk factor for type 2 diabetes ${ }^{29}$. There are few studies that have examined trajectories of blood lipid trajectories and their relationship to chronic diseases ${ }^{30,31}$. Our study found that higher values of serum HDL over time were associated with reduced risk of diabetes onset, while higher values of serum non-HDL and triglycerides were associated with increased risk of disease. To recap our study's inclusion criteria, there were two strict observational data requirements for a patient to be included in the analyses in this paper. First, each patient had to meet or exceed the LEADR criterion that required a specific number of visits to one of the 9 health systems included in LEADR over the specific time period being observed. Second was the requirement for each patient to have an adequate number of clinical values recorded in their EHR to allow for the generation of multiple class trajectories for that patient over the specific time period being observed. Patients who were excluded from these analyses lacked sufficient EHR data for one or both of these data requirements, and therefore were not included in the analyses. The resulting total population of 94,565 that met these observational criteria are thereby a welldefined sample of patients from the larger LEADR cohort who had adequate health system visits and records of clinical measurements.

We are aware that differential missing/attrition is common in EHR data, and as a result, bias can often be introduced into analysis if the estimates, including trajectory group size and membership, are not well delineated initially. Missing data can affect group sizes change over time, which in turn has important implications for using the model parameter estimates to make population-level projections. We recognize that in future analysis, applying an extension of group-based trajectory modeling that would have the ability to address missing data, non-random attrition, or truncation of data due to specific events, such as death, is warranted. Additionally, building on the analytic relationships discovered in this study, further investigation is necessary into the interplay of risk factors for type 2 diabetes, to better fully understand the effect of various combinations of trajectories on progression to disease. For example, future research could investigate how the risks associated with the trajectories are different among those with prediabetes.

Despite this study's limitations, our analyses indicate having trend data for risk factors adds an additional perspective that takes advantage of analyzing longitudinal data that provides insights to assessing risk for disease moving well beyond the contribution of a single BMI measurement.

\section{Acknowledgments}

This project was solely funded by the Centers for Disease Control and Prevention under contract HHSD200201587699 to Westat Inc. The findings and conclusions in this report are those of the authors and do not necessarily represent the official position of the Centers for Disease Control and Prevention or the National Institutes of Health. The article contents have not been presented elsewhere. The authors declare that they have no conflict of interest. No financial disclosures were reported by the authors of this paper.

Study concept, data collection, analysis, and manuscript preparation and review completed by Howard A. Fishbein, DrPH, MPH; Qilu Yu, PhD; Maurice Johnson, MPH; Rebecca Birch, MPH; Holly Sawyer, BA; Sunitha M. Mathew, MS; Lori Merrill, MS. and Wilson Pace, MD. Study concept and manuscript review completed by Russ Mardon, PhD and Dan Bauer, PhD. Programming support, data collection and harmonization provided by Keith Umbel, BS.

\section{Conflict of Interest}

All authors approved the manuscript before submission and declared no financial disclosures or conflicts of interest.

\section{References}

1. American Diabetes Association. Economic costs of diabetes in the U.S. in 2017. Diabetes Care 2018; 41(5): 917-928.

2. Centers for Disease Control and Prevention. National Diabetes Statistics Report: Estimates of Diabetes and Its Burden in the United States, 2017. Atlanta, GA, U.S. Department of Health and Human Services, Centers for Disease Control and Prevention.

3. McKeever Bullard K, Cowie C, Lessem S, et al. Prevalence of Diagnosed Diabetes in Adults by Diabetes Type - United States 2016. MMWR Morb Mortal Wkly Rep. 2018; 67(12): 359-361.

4. National Institutes of Health NIDDK. Risk Factors for Type 2 Diabetes. 2016. https://www.niddk.nih.gov/health-information/diabetes/ overview/risk-factors-type-2-diabetes

5. Alharbi KK, Abudawood M, Khan IA. Amino-acid amendment of arginine-325-tryptophan in rs13266634 genetic polymorphism studies of the SLC30A8 gene with type 2 diabetes-mellitus patients featuring a positive family history in the Saudi population. Journal of King Saud University-Science. 2021; 33(1): 101258.

6. Mozaffarian D, Kamineni A, Carnethon M, et al. Lifestyle Risk Factors and New-Onset Diabetes Mellitus in Older Adults. Arch Intern Med. 2009; 169(8): 798-807.

7. Wu Y, Ding Y, Tanaka Y, et al. Risk Factors Contributing to Type 2 Diabetes and Recent Advances in the Treatment and Prevention. Int J Med Sci. 2014; 11(11): 1185-1200.

8. Wang K, Chen X, Gerke TA, et al. BMI trajectories and risk of overall and grade-specific prostate cancer: An observational cohort study among men seen for prostatic conditions. Cancer medicine. 2018; $7(10)$ : 5272-5280

9. Edwards MK, Addoh O, Sng E, et al. Physical activity, body mass index and waist circumference change, and normal-range glycated hemoglobin on incident diabetes: Jackson Heart Study. Postgrad Med. 2017; 129(8): 842-848. 
10. Hu H, Nagahama S, Nanri A, et al. Duration and degree of weight change and risk of incident diabetes: Japan Epidemiology Collaboration on Occupational Health Study. Prev Med. 2017; 96: 118-123.

11. Walsh EI, Shaw J, Cherbuin N. Trajectories of BMI change impact glucose and insulin metabolism. Nutr Metab Cardiovasc Dis. 2018; 28(3): 243-251.

12. Heianza Y, Arase Y, Kodama S, et al. Trajectory of body mass index before the development of type 2 diabetes in Japanese men: Toranomon Hospital Health Management Center Study 15. J Diabetes Investig. 2015; 6(3): 289-294.

13. Stokes A, Collins JM, Grant BF, et al. Obesity progression between young adulthood and midlife and incident diabetes: A retrospective cohort study of U.S. adults. Diabetes Care. 2018; 41(5): 1025-1031.

14. Chiu CJ, Li SL, Wu CH, et al. BMI Trajectories as a Harbinger of Pre-Diabetes or Underdiagnosed Diabetes: an 18-Year Retrospective Cohort Study in Taiwan. J Gen Intern Med. 2016; 31(10): 1156-63.

15. Eriksson JG, Kajantie E, Lampl M, et al. Trajectories of body mass index amongst children who develop type 2 diabetes as adults. Journal of internal medicine. 2015; 278(2): 219-226.

16. Kuwahara K, Honda T, Nakagawa T, et al. Body mass index trajectory patterns and changes in visceral fat and glucose metabolism before the onset of type 2 diabetes. Scientific reports. 2017; 7: 43521.

17. Peter RS, Keller F, Klenk J, et al. Body mass trajectories, diabetes mellitus, and mortality in a large cohort of Austrian adults. Medicine (Baltimore). 2016; 95(49): e5608.

18. Vistisen D, Witte DR, Tabák AG, et al. Patterns of obesity development before the diagnosis of type 2 diabetes: the Whitehall II cohort study. PLoS medicine. 2014; 11(2): e1001602.

19. Færch K, Witte DR, Tabák AG, et al. Trajectories of cardiometabolic risk factors before diagnosis of three subtypes of type 2 diabetes: a post-hoc analysis of the longitudinal Whitehall II cohort study. The Lancet Diabetes \& Endocrinology. 2013; 1(1): 43-51.

20. Habiba NM, Fulda KG, Basha R, et al. Correlation of Lipid Profile and Risk of Developing Type 2 Diabetes Mellitus in 10-14 Year Old Children. Cellular Physiology and Biochemistry. 2016; 39(5): 16951704.
21. Rhee EJ, Han K, Ko SH, et al. Increased risk for diabetes development in subjects with large variation in total cholesterol levels in 2,827,950 Koreans: a nationwide population-based study. PloS one. 2017; 12(5): e0176615.

22. Fishbein HA, Birch RJ, Mathew SM, et al. The Longitudinal Epidemiologic Assessment of Diabetes Risk (LEADR): Unique 1.4 M patient Electronic Health Record cohort. Healthcare. 2020; 8(4): 100458.

23. Reinecke J, Seddig D. Growth mixture models in longitudinal research. Adv Stat Anal 2011; 95(4): 415-434.

24. Makadia R, Ryan PB. Transforming the Premier Perspective Hospital Database into the Observational Medical Outcomes Partnership (OMOP) Common Data Model. EGEMS (Wash DC) 2014; 2(1): 1110.

25. Chopra HK, Ram C. Recent Guidelines for Hypertension. Circulation Research. 2019; 124(7): 984-986.

26. Grundy SM, Stone NJ, Bailey AL, et al. 2018 AHA/ACC/AACVPR/ AAPA/ABC/ACPM/ADA/AGS/APhA/ASPC/NLA/PCNA Guideline on the Management of Blood Cholesterol: A Report of the American College of Cardiology/American Heart Association Task Force on Clinical Practice Guidelines. Circulation. 2019; 139(25): e1082-e1143

27. Lindström J, Ilanne-Parikka P, Peltonen M, et al. Finnish Diabetes Prevention Study Group Sustained reduction in the incidence of type 2 diabetes by lifestyle intervention: follow-up of the Finnish Diabetes Prevention Study. Lancet. 2006; 368(9548): 1673-9.

28. Knowler WC, Barrett-Connor E, Fowler SE, et al. Reduction in the incidence of type 2 diabetes with lifestyle intervention or metformin. The New England journal of medicine. 2002; 346(6): 393-403.

29. Emdin CA, Anderson SG, Woodward M, et al. Usual blood pressure and risk of new-onset diabetes: Evidence from 4.1 million adults and a meta-analysis of prospective studies. J Am Coll Cardiol. 2015: 66(14): 1552-1562.

30. Duncan S, Vasan RS, Xanthakis V. Trajectories of blood lipid concentrations over the adult life course and risk of cardiovascular disease and all-case mortality: Observations from the Framingham study over 35 years. J Am Heart Assoc. 2019; 8(11): e011433.

31. Wagner M, Helmer C, Tzourio C, et al. Evaluation of the concurrent trajectories of cardiometabolic risk factors in the 14 years before dementia. JAMA Psychiatry. 2018; 75(10): 1033-1042. 


\section{SUPPLEMENTAL MATERIAL}

Table S1: Number and Percentage of LEADR Cohort Patients in Each Trajectory Class* of Percentage Change in BMI by Sex and Age Group

\begin{tabular}{|c|c|c|c|c|c|c|c|c|c|c|c|c|}
\hline \multirow{4}{*}{$\begin{array}{c}\text { Trajectory } \\
\text { Classes }\end{array}$} & \multicolumn{12}{|c|}{ Total $(\mathrm{N}=824,043)$} \\
\hline & \multicolumn{6}{|c|}{ Female $(N=499,846)$} & \multicolumn{6}{|c|}{ Male $(\mathrm{N}=324,197)$} \\
\hline & \multicolumn{2}{|c|}{ Age 18-44 } & \multicolumn{2}{|c|}{ Age 45-64 } & \multicolumn{2}{|c|}{ Age 65+ } & \multicolumn{2}{|c|}{ Age 18-44 } & \multicolumn{2}{|c|}{ Age 45-64 } & \multicolumn{2}{|c|}{ Age 65+ } \\
\hline & $\mathbf{N}$ & $\%$ & $\mathbf{N}$ & $\%$ & $\mathbf{N}$ & $\%$ & $\mathbf{N}$ & $\%$ & $\mathbf{N}$ & $\%$ & $\mathbf{N}$ & $\%$ \\
\hline Total & 228,146 & 100 & 190,350 & 100 & 81,350 & 100 & 122,662 & 100 & 139,125 & 100 & 62,410 & 100 \\
\hline 1 & 190,850 & 83.7 & 165,951 & 87.2 & 67,732 & 83.3 & 104,564 & 85.2 & 122,020 & 87.7 & 53,509 & 85.7 \\
\hline 2 & 10,668 & 4.7 & 9,269 & 4.9 & 5,464 & 6.7 & 4,856 & 4.0 & 6,656 & 4.8 & 3,561 & 5.7 \\
\hline 3 & 19,173 & 8.4 & 10,357 & 5.4 & 6,060 & 7.4 & 9,773 & 8.0 & 7,311 & 5.3 & 3,830 & 6.1 \\
\hline 4 & 2,704 & 1.2 & 2,128 & 1.1 & 1,639 & 2.0 & 1,231 & 1.0 & 1,527 & 1.1 & 800 & 1.3 \\
\hline 5 & 4,751 & 2.1 & 2645 & 1.4 & 455 & 0.6 & 2,238 & 1.8 & 1,611 & 1.2 & 710 & 1.1 \\
\hline
\end{tabular}

* The study follow-up period is 5 years. BMI calculated as $\mathrm{kg} / \mathrm{m}^{2}$.

Table S2: Number and Percentage of LEADR Cohort Patients in Each Trajectory Class of Systolic Blood Pressure (SBP), Diastolic Blood Pressure (DBP), High-density Lipoprotein (HDL), non-HDL, and Triglyceride Class*

\begin{tabular}{|c|c|c|c|c|c|c|c|c|c|c|c|c|c|c|c|c|c|c|}
\hline \multirow{3}{*}{$\begin{array}{c}\text { Trajectory } \\
\text { Classes }\end{array}$} & \multicolumn{6}{|c|}{$\begin{array}{l}\text { Systolic Blood Pressure } \\
\text { (N=955,128) }\end{array}$} & \multicolumn{6}{|c|}{$\begin{array}{l}\text { Diastolic Blood Pressure } \\
\qquad(\mathrm{N}=957,491)\end{array}$} & \multirow{2}{*}{\multicolumn{2}{|c|}{$\left.\begin{array}{c}\text { HDL } \\
(\mathrm{N}=\mathbf{3 0 0 , 1 3 7 )}\end{array}\right)$}} & \multirow{2}{*}{\multicolumn{2}{|c|}{$\begin{array}{c}\begin{array}{c}\text { Non-HDL } \\
(\mathrm{N}=267,553)\end{array} \\
\text { All Ages }\end{array}$}} & \multirow{2}{*}{\multicolumn{2}{|c|}{ 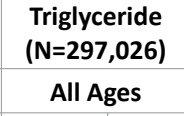 }} \\
\hline & \multicolumn{2}{|c|}{ Age 18-44 } & \multicolumn{2}{|c|}{ Age 45-64 } & \multicolumn{2}{|c|}{$65+$} & \multicolumn{2}{|r|}{$8-44$} & \multicolumn{2}{|c|}{ Age 45-64 } & \multicolumn{2}{|c|}{ e $65+$} & & & & & & \\
\hline & $\mathbf{N}$ & $\%$ & & $\ldots$ & $N$ & $\%$ & 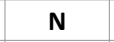 & $\%$ & $\ldots$ & $\%$ & 1. & $\%$ & 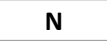 & $\%$ & $\mathbf{N}$ & $\%$ & $\mathbf{N}$ & $\%$ \\
\hline Total & 421,327 & 100.0 & 364,216 & 100.0 & 169,582 & 100.0 & 422,348 & 100.0 & 365,230 & 100.0 & 169,913 & 100.0 & 300,137 & 100.0 & 267,553 & 100.0 & 7,026 & 100.0 \\
\hline 1 & & & & 89.4 & & 87.8 & 896 & 85.7 & 35 & 97.0 & & .5 & 537 & 66.8 & & 3 & & 1.6 \\
\hline 2 & & & & & & & & 13.7 & & & & & & 19.2 & 60 & 11.2 & & 33.0 \\
\hline $\mathrm{J}$ & $8<0$ & 3.1 & 3,86 & 5.2 & 113 & 0.0 & 518 & 0. & 6196 & 1.1 & 2408 & 1.4 & 36326 & 12.1 & 3988 & 1.5 & 9685 & 6.6 \\
\hline 4 & - & - & - & - & - & - & - & - & - & - & - & - & 5563 & 1.9 & - & - & 5431 & 8.6 \\
\hline 5 & - & - & - & - & - & - & - & - & - & - & - & - & - & - & - & - & 6495 & 2.2 \\
\hline
\end{tabular}

* Study period is 5 years. Blood pressures measured $\mathrm{mm} \mathrm{Hg}$, lipids measured $\mathrm{mg} / \mathrm{dL}$. 